\title{
A Novel Approach to Neutrosophic Soft Rough Set under Uncertainty
}

\author{
Ashraf Al-Quran 1®, Nasruddin Hassan ${ }^{2, *}$, and Emad Marei ${ }^{3,+}$ \\ 1 Department of Mathematics, Faculty of Science, Jerash University, Jerash 26150, Jordan; a.quraan@jpu.edu.jo \\ 2 Faculty of Science and Technology, School of Mathematical Sciences, Universiti Kebangsaan Malaysia, \\ UKM Bangi Selangor 43600, DE, Malaysia \\ 3 Department of Mathematics, Faculty of Science and Art, Shaqra University, Shaqra 11961, Saudi Arabia; \\ awajan@su.edu.sa \\ * Correspondence: nas@ukm.edu.my; Tel.: +60-3-8921-3710 \\ + Deceased 13 July 2018.
}

Received: 5 January 2019; Accepted: 25 February 2019; Published: 15 March 2019

\begin{abstract}
To handle indeterminate and incomplete data, neutrosophic logic/set/probability were established. The neutrosophic truth, falsehood and indeterminacy components exhibit symmetry as the truth and the falsehood look the same and behave in a symmetrical way with respect to the indeterminacy component which serves as a line of the symmetry. Soft set is a generic mathematical tool for dealing with uncertainty. Rough set is a new mathematical tool for dealing with vague, imprecise, inconsistent and uncertain knowledge in information systems. This paper introduces a new rough set model based on neutrosophic soft set to exploit simultaneously the advantages of rough sets and neutrosophic soft sets in order to handle all types of uncertainty in data. The idea of neutrosophic right neighborhood is utilised to define the concepts of neutrosophic soft rough (NSR) lower and upper approximations. Properties of suggested approximations are proposed and subsequently proven. Some of the NSR set concepts such as NSR-definability, NSR-relations and NSR-membership functions are suggested and illustrated with examples. Further, we demonstrate the feasibility of the newly rough set model with decision making problems involving neutrosophic soft set. Finally, a discussion on the features and limitations of the proposed model is provided.
\end{abstract}

Keywords: decision making; membership function; neutrosophic set; neutrosophic soft rough; relations; rough set approximation; soft set

\section{Introduction}

The limitation of deterministic research is currently recognized in areas of management, social sciences, operations research and economics. Uncertain theories such as probability, fuzzy sets [1], intuitionistic fuzzy sets [2], vague sets [3] and theory of interval mathematics [4] are applied in realms which are ambiguous and uncertain.

Rough set theory, initiated by Pawlak [5], is an effective mathematical tool to the vague and imperfect knowledge. Rough set expresses vagueness by bounded region of a set, which can be interpreted as using the vagueness of Frege's idea. Pawlak argued that any vague concept can be replaced by the lower and upper approximations of precise sets using an equivalence relation. In the real application, the equivalence relation is a very stringent condition which limits the applications of rough sets in the real world. For this reason, by replacing the equivalence relation with covering, similarity, tolerance, preference, dominance relations, and different neighborhood operators, various kinds of rough set generalizations model were proposed [6-11].

A soft set is a set-valued map defined by Molodtsov [12], to approximately describe objects using several parameters. Maji et al. [13] applied the theory of soft set to solve decision making problems with 
the help of rough mathematics. The adequate parametrization capabilities of soft set theory and the lack of such capabilities in the fuzzy set served as the motivation to introduce the fuzzy soft sets [14].

Feng et al. [15] proved properties of soft rough set model. Smarandache [16,17] proposed neutrosophic set to handle problems containing imprecise, incomplete, uncertain and indeterminate data. Neutrosophic sets progressed rapidly to neutrosophic oversets, neutrosophic undersets, neutrosophic offsets [18], neutrosophic cubic sets [19], neutrosophic and generalised neutrosophic soft sets [20-22], neutrosophic rough sets [23-26], neutrosophic vague sets [27,28] and complex neutrosophic sets [29-32].

We will propose an approach to neutrosophic soft rough set and show that the traditional rough approach is a special case of our approach. Furthermore, we will study the neutrosophic soft rough approximations and apply them to decision making. The paper is organized into seven sections. Section 2 provides literature review. In Section 3, the concept of neutrosophic right neighborhood is defined. This section further defines neutrosophic soft rough set approximations. Properties of NSR-lower and NSR-upper approximations are included along with supported proofs and illustrated examples. Section 4 delves into neutrosophic soft rough set and generalization of rough concepts. NSR-set concepts include neutrosophic soft rough (NSR) definability, neutrosophic soft rough (NSR)-membership function, neutrosophic soft rough (NSR)-membership relations, neutrosophic soft rough (NSR)-inclusion relations and neutrosophic soft rough (NSR)-equality relations. Properties of these concepts are proven and examples provided. Section 5 provides an application of the proposed neutrosophic soft rough model on decision making. In Section 6, we conduct a discussion about the features and limitations of the proposed model by making a comparison with the existing models. In the final section, we outline future work and draw conclusions to this work.

\section{Preliminaries}

We start by reviewing the concepts of rough set, neutrosophic set and soft set.

Pawlak considered the set $X$ with the equivalence relation $E$ and called the pair $(X, E)$ as a Pawlak approximation space. Then he assigned two operations (lower and upper approximations) to any vague subset $M \subseteq X$. These operations process some information connected with the relationship $E$ and they are analogous with Kuratowski's operations, which are generated by the closure and the completion.

The lower, upper and boundary approximations are defined as follows.

Definition 1 ([5]). Let $E$ be an equivalence relation on a universe $X$ and $M \subseteq X$. Then the pair $(X, E)$ is referred to as a Pawlak approximation space. The lower, upper and boundary approximations of $M$ are defined as follows.

$$
\begin{gathered}
\underline{E}(M)=\cup\left\{[x]_{E}:[x]_{E} \subseteq M\right\}, \\
\bar{E}(M)=\cup\left\{[x]_{E}:[x]_{E} \cap M \neq \phi\right\}, \\
B N D_{E}(M)=\bar{E}(M)-\underline{E}(M) .
\end{gathered}
$$

where $[x]_{E}=\left\{x^{\prime} \in X: E(x)=E\left(x^{\prime}\right)\right\}$.

Definition 2 ([5]). Let $A=(X, E)$ be an approximation space and let $M \subset X$. By the accuracy of approximation of $M$ in $A$ we mean the number

$$
\alpha_{E}(M)=\frac{|\underline{E}(M)|}{|\bar{E}(M)|}, \bar{E}(M) \neq \varnothing .
$$

Obviously, $0 \leq \alpha_{E}(M) \leq 1$. If $\bar{E}(M)=\underline{E}(M)$, then $M$ is crisp (exact) set, with respect to $E$, otherwise $M$ is rough set. 
The following proposition lists the properties of Pawlak's approximations.

Proposition 1 ([5]). For every $M, Y \subset X$ and every approximation space $A=(X, E)$ the following properties hold:

(i) $\underline{E}(M) \subseteq M \subseteq \bar{E}(M)$.

(ii) $\underline{E}(\phi)=\phi=\bar{E}(\phi)$ and $\underline{E}(X)=X=\bar{E}(X)$.

(iii) $\bar{E}(M \cup Y)=\bar{E}(M) \cup \bar{E}(Y)$.

(iv) $\underline{E}(M \cap Y)=\underline{E}(M) \cap \underline{E}(Y)$.

(v) $M \subseteq Y$, then $\underline{E}(M) \subseteq \underline{E}(Y)$ and $\bar{E}(M) \subseteq \bar{E}(Y)$.

(vi) $\underline{E}(M \cup Y) \supseteq \underline{E}(M) \cup \underline{E}(Y)$.

(vii) $\bar{E}(M \cap Y) \subseteq \bar{E}(M) \cap \bar{E}(Y)$.

(viii) $\underline{E}\left(M^{c}\right)=[\bar{E}(M)]^{c}$, where $M^{c}$ is the complement of $M$.

(ix) $\bar{E}\left(M^{c}\right)=[\underline{E}(M)]^{c}$.

(x) $\underline{E}(\underline{E}(M))=\bar{E}(\underline{E}(M))=\underline{E}(M)$.

(xi) $\bar{E}(\bar{E}(M))=\underline{E}(\bar{E}(M))=\bar{E}(M)$.

Definition 3 ([33]). An information system is a quadruple $I S=(U, A, V, f)$, where $U$ is a non-empty finite set of objects, $A$ is a non-empty finite set of attributes, $V=\cup\left\{V_{e}, e \in A\right\}, V_{e}$ is the set of values of attribute $e$, and $f: U \times A \rightarrow V$, is called an information (knowledge) function.

Definition 4 ([12]). Let $X$ be an initial universe set, $E$ be a set of parameters, $A \subseteq E$ and let $P(X)$ denote the power set of $X$. Then, a pair $K=(L, A)$ is called a soft set over $X$, where $L$ is a mapping given by $L: A \rightarrow P(X)$. In other words, a soft set over $X$ is a parameterized family of subsets of $X$. For $a \in A, L(a)$ may be considered as the set of a-approximate elements of $K$.

The neutrosophic set was defined by Smarandache below.

Definition 5 ([17]). A neutrosophic set $N$ on the universe of discourse $X$ is defined as

$$
\begin{gathered}
N=\left\{\left\langle n, T_{N}(n), I_{N}(n), F_{N}(n)\right\rangle: n \in X\right\}, \text { where } \\
-0 \leq T_{N}(n)+I_{N}(n)+F_{N}(n) \leq 3^{+}, \text {where } \\
T, I, F: X \longrightarrow]^{-} 0 ; 1^{+}[.
\end{gathered}
$$

\section{Neutrosophic Soft Rough Set Approximations (NSR-Set Approximations)}

In this section, we give a definition of neutrosophic soft set (NSS in short) with an illustrative example. We will introduce and provide examples of NSR-lower and NSR-upper approximations.

Definition 6. Let a universe $X, E$ the parameter set and $A \subseteq E$. A neutrosophic soft set $H$ over $X$ is a neutrosophic set valued function from $A$ to $P(X)$. It can be written as

$$
H=\left\{\left(a,<x, T_{H(a)}(x), I_{H(a)}(x), F_{H(a)}(x)>: x \in X\right): a \in E\right\},
$$

where $P(X)$ denotes the power neutrosophic set of $X$.

In other words, the neutrosophic soft set $H$ is a parameterized family of neutrosophic subsets of $X$. For any parameter $a, H(a)$ is referred as the neutrosophic value set of parameter $a$.

The example below will convey the meaning of neutrosophic soft set.

Example 1. Let $X$ be a set of houses and $E$ be a set of parameters (or qualities). Consider $E=\{$ cheap, beautiful, green surrounding, spacious $\}$. To define an (NSS) means to point out cheap houses, beautiful houses and so 
on. If there are five houses in $X$, where, $X=\left\{x_{1}, x_{2}, x_{3}, x_{4}, x_{5}\right\}$ and the set of parameters $A=\left\{a_{1}, a_{2}, a_{3}, a_{4}\right\}$, where $A \subset E$, and each $a_{i}$ is a specific property for houses: $a_{1}$ stands for (cheap), $a_{2}$ stands for (beautiful), $a_{3}$ stands for (green surrounding), $a_{4}$ stands for (spacious).

An (NSS) can be represented as in Table 1 , such that the entries are $h_{i j}$ corresponding to the house $x_{i}$ and the parameter $a_{j}$, where $h_{i j}=$ (true membership value of $x_{i}$, indeterminacy-membership value of $x_{i}$, falsity membership value of $\left.x_{i}\right)$ in $H\left(a_{j}\right)$. Table 1 , represents the (NSS) $(H, A)$ as follows.

Table 1. Tabular representation of the neutrosophic soft set (NSS) $(H, A)$.

\begin{tabular}{ccccc}
\hline $\boldsymbol{X}$ & $\boldsymbol{a}_{\boldsymbol{1}}$ & $\boldsymbol{a}_{\mathbf{2}}$ & $\boldsymbol{a}_{3}$ & $\boldsymbol{a}_{\mathbf{4}}$ \\
\hline$x_{1}$ & $(0.6,0.6,0.2)$ & $(0.8,0.4,0.3)$ & $(0.7,0.4,0.3)$ & $(0.8,0.6,0.4)$ \\
$x_{2}$ & $(0.4,0.6,0.6)$ & $(0.6,0.2,0.4)$ & $(0.6,0.4,0.3)$ & $(0.7,0.6,0.6)$ \\
$x_{3}$ & $(0.6,0.4,0.2)$ & $(0.8,0.1,0.3)$ & $(0.7,0.2,0.5)$ & $(0.7,0.6,0.4)$ \\
$x_{4}$ & $(0.6,0.3,0.3)$ & $(0.8,0.2,0.2)$ & $(0.5,0.2,0.6)$ & $(0.7,0.5,0.6)$ \\
$x_{5}$ & $(0.8,0.2,0.3)$ & $(0.8,0.3,0.2)$ & $(0.7,0.3,0.4)$ & $(0.9,0.5,0.7)$ \\
\hline
\end{tabular}

In the following, we define the concept of the neutrosophic right neighborhood.

Definition 7. Let $X$ be a universal set and $\Gamma$ be the power set of $X$. Let $(H, A)$ be an (NSS) on $X$, and $\omega=A \times X$. Let $S$ be a mapping given by

$$
S: \omega \rightarrow \Gamma,
$$

where $S_{a}(x)=S(a, x)=\left\{x_{i} \in X: T_{a}\left(x_{i}\right) \geq T_{a}(x)\right.$ and $I_{a}\left(x_{i}\right) \geq I_{a}(x)$ and $\left.F_{a}\left(x_{i}\right) \leq F_{a}(x)\right\}$.

Then for any element $x \in X, S_{a}(x)$ is called a neutrosophic right neighborhood, with respect to $a \in A$.

Definition 8. Let $X$ be a universal set and $(H, A)$ be an (NSS) on $X$. Then for all $x \in X$ and $a \in A$, the family of all neutrosophic right neighborhoods is defined as follows.

$$
\psi=\left\{S_{a}(x): x \in X, a \in A\right\} .
$$

The example below conveys the meaning of neutrosophic right neighborhoods.

Example 2. We can deduce the statements below from Example 1.

$S_{a_{1}}\left(x_{1}\right)=S_{a_{2}}\left(x_{1}\right)=S_{a_{3}}\left(x_{1}\right)=S_{a_{4}}\left(x_{1}\right)=\left\{x_{1}\right\}$,

$S_{a_{1}}\left(x_{2}\right)=S_{a_{3}}\left(x_{2}\right)=\left\{x_{1}, x_{2}\right\}, S_{a_{2}}\left(x_{2}\right)=\left\{x_{1}, x_{2}, x_{4}, x_{5}\right\}, S_{a_{4}}\left(x_{2}\right)=\left\{x_{1}, x_{2}, x_{3}\right\}$,

$S_{a_{1}}\left(x_{3}\right)=S_{a_{4}}\left(x_{3}\right)=\left\{x_{1}, x_{3}\right\}, S_{a_{2}}\left(x_{3}\right)=\left\{x_{1}, x_{3}, x_{4}, x_{5}\right\}, S_{a_{3}}\left(x_{3}\right)=\left\{x_{1}, x_{3}, x_{5}\right\}$,

$S_{a_{1}}\left(x_{4}\right)=\left\{x_{1}, x_{3}, x_{4}\right\}, S_{a_{2}}\left(x_{4}\right)=\left\{x_{4}, x_{5}\right\}, S_{a_{3}}\left(x_{4}\right)=X, S_{a_{4}}\left(x_{4}\right)=\left\{x_{1}, x_{2}, x_{3}, x_{4}\right\}$,

$S_{a_{1}}\left(x_{5}\right)=S_{a_{2}}\left(x_{5}\right)=S_{a_{4}}\left(x_{5}\right)=\left\{x_{5}\right\}, S_{a_{3}}\left(x_{5}\right)=\left\{x_{1}, x_{5}\right\}$.

It follows that, $\psi=\left\{\left\{x_{1}\right\},\left\{x_{5}\right\},\left\{x_{1}, x_{2}\right\},\left\{x_{1}, x_{3}\right\},\left\{x_{1}, x_{5}\right\},\left\{x_{4}, x_{5}\right\},\left\{x_{1}, x_{2}, x_{3}\right\},\left\{x_{1}, x_{3}, x_{4}\right\}\right.$, $\left.\left\{x_{1}, x_{3}, x_{5}\right\},\left\{x_{1}, x_{2}, x_{3}, x_{4}\right\},\left\{x_{1}, x_{2}, x_{4}, x_{5}\right\},\left\{x_{1}, x_{3}, x_{4}, x_{5}\right\}, X\right\}$.

Proposition 2. Let $(H, A)$ be an (NSS) on a universe $X, \psi$ is the parameterised family of all neutrosophic right neighborhoods and $R_{a}: X \rightarrow \psi, R_{a}(x)=S_{a}(x)$. Then the statements below hold.

(i) $R_{a}$ is reflexive relation.

(ii) $R_{a}$ is transitive relation.

Proof. Let $\left\langle x_{1}, T_{a}\left(x_{1}\right), I_{a}\left(x_{1}\right), F_{a}\left(x_{1}\right)\right\rangle,\left\langle x_{2}, T_{a}\left(x_{2}\right), I_{a}\left(x_{2}\right), F_{a}\left(x_{2}\right)\right\rangle$ and $\left\langle x_{3}, T_{a}\left(x_{3}\right), I_{a}\left(x_{3}\right), F_{a}\left(x_{3}\right)\right\rangle \in$ $(H, A)$. Then,

(i) Obviously, for all $i=1,2,3, T_{a}\left(x_{i}\right) \geq T_{a}\left(x_{i}\right), I_{a}\left(x_{i}\right) \geq I_{a}\left(x_{i}\right), F_{a}\left(x_{i}\right) \leq F_{a}\left(x_{i}\right)$ Hence, for every $a \in A$, $x_{i} \in S_{a}\left(x_{i}\right)$ and $x_{i} R_{a} x_{i}$ and thus $R_{a}$ is reflexive relation. 
(ii) Let $x_{1} R_{a} x_{2}$ and $x_{2} R_{a} x_{3}$. Then, $x_{2} \in S_{a}\left(x_{1}\right)$ and $x_{3} \in S_{a}\left(x_{2}\right)$. Hence, $T_{a}\left(x_{2}\right) \geq T_{a}\left(x_{1}\right), I_{a}\left(x_{2}\right) \geq I_{a}\left(x_{1}\right)$, $F_{a}\left(x_{2}\right) \leq F_{a}\left(x_{1}\right), T_{a}\left(x_{3}\right) \geq T_{a}\left(x_{2}\right), I_{a}\left(x_{3}\right) \geq I_{a}\left(x_{2}\right)$ and $F_{a}\left(x_{3}\right) \leq F_{a}\left(x_{2}\right)$. Consequently, wa have $T_{a}\left(x_{3}\right)$ $\geq T_{a}\left(x_{1}\right), I_{a}\left(x_{3}\right) \geq I_{a}\left(x_{1}\right)$ and $F_{a}\left(x_{3}\right) \leq F_{a}\left(x_{1}\right)$. It follows that $x_{3} \in S_{a}\left(x_{1}\right)$ and $x_{1} R_{a} x_{3}$ and thus $R_{a}$ is transitive relation.

Note that $R_{a}$ in Proposition 2 may not necessarily be symmetric as shown below.

Example 3. From Example 2, we have, $S_{a_{1}}\left(x_{1}\right)=\left\{x_{1}\right\}$ and $S_{a_{1}}\left(x_{3}\right)=\left\{x_{1}, x_{3}\right\}$. Hence, $\left(x_{3}, x_{1}\right) \in R_{a_{1}}$ but $\left(x_{1}, x_{3}\right) \notin R_{a_{1}}$. Thus $R_{a}$ is not symmetric relation.

We define the neutrosophic soft rough lower and upper approximations below.

Definition 9. Let $(H, A)$ be an (NSS) on a universe $X$, with $\psi$ being the family of all neutrosophic right neighborhoods. The neutrosophic soft lower and neutrosophic soft upper approximations of any subset $M$ based on $\psi$, respectively, are

$$
\begin{gathered}
N R_{*} M=\cup\{Y \in \psi: Y \subseteq M\}, \\
N R^{*} M=\cup\{Y \in \psi: Y \cap M \neq \varnothing\} .
\end{gathered}
$$

$N R_{*} M$ and $N R^{*} M$ can be referred as neutrosophic soft rough approximations of $M$ (NSR-set approximations) with respect to $A$.

Remark 1. For any considered set $M$ in an (NSS) $(H, A)$, the sets $\operatorname{Pos}_{N R} M=N R_{*} M, N e g_{N R} M=$ $\left[N R^{*} M\right]^{c}, b_{N R} M=N R^{*} M-N R_{*} M$ are called the NSR-positive, NSR-negative and NSR-boundary regions of a considered set $M$, respectively. The meaning of $\operatorname{Pos}_{N R} M$ is the set of all elements, which are surely belonging to $M, N e g_{N R} M$ is the set of all elements, which do not belong to $M$ and $b_{N R} M$ is the elements of $M$, not determined by $(H, A)$.

The proposition below lists the properties of neutrosophic soft rough approximations.

Proposition 3. Let $(H, A)$ be an (NSS) on a universe $X$, and let $M, Z \subseteq X$. Then the following properties hold.

(i) $N R_{*} M \subseteq M \subseteq N R^{*} M$.

(ii) $N R_{*} \varnothing=N R^{*} \varnothing=\varnothing$.

(iii) $N R_{*} X=N R^{*} X=X$.

(iv) $M \subseteq Z \Rightarrow N R_{*} M \subseteq N R_{*} Z$.

(v) $M \subseteq Z \Rightarrow N R^{*} M \subseteq N R^{*} Z$.

(vi) $N R_{*}(M \cap Z) \subseteq N R_{*} M \cap N R_{*} Z$.

(vii) $N R_{*}(M \cup Z) \supseteq N R_{*} M \cup N R_{*} Z$.

(viii) $N R^{*}(M \cap Z) \subseteq N R^{*} M \cap N R^{*} Z$.

(ix) $N R^{*}(M \cup Z)=N R^{*} M \cup N R^{*} Z$.

Proof. (i) From Definition 9, we can deduce that $N R_{*} M \subseteq M$. In addition, let $x \in M$, but $R_{a}$ defined in Proposition 2 is reflexive relation. For all $a \in A$, there exists $S_{a}(x)$ such that $x \in S_{a}(x)$ and there exists $Y \in \psi$ such that $Y \cap M \neq \varnothing$. Hence, $x \in N R^{*} M$. Thus $N R_{*} M \subseteq M \subseteq N R^{*} M$.

(ii) Proof of (ii) follows directly from Definition 9.

(iii) From property (i), we have $X \subseteq N R^{*} X$. Since $X$ is the universe set $N R^{*} X=X$. From Definition 9, we have $N R_{*} X=\cup\{Y \in \psi: Y \subseteq X\}$, but for all $x \in X$, there exists $S_{a}(x) \in \psi$ such that $x$ $\in S_{a}(x) \subseteq X$. Hence, $N R_{*} X=X$. Thus $N R_{*} X=N R^{*} X=X$.

(iv) Let $M \subseteq Z$ and $x \in N R_{*} M$. There exists $Y \in \psi$ such that $x \in Y \subseteq M$. However, $M \subseteq Z$, thus $x \in Y \subseteq Z$. Hence, $x \in N R_{*} Z$. Consequently, $N R_{*} M \subseteq N R_{*} Z$. 
(v) Let $M \subseteq Z$ and $x \in N R^{*} M$. There exists $Y \in \psi$ such that $x \in Y, Y \cap M \neq \varnothing$. However, $M \subseteq Z$, thus $Y \cap Z \neq \varnothing$. Hence, $x \in N R^{*} Z$. Thus $N R^{*} M \subseteq N R^{*} Z$.

(vii) Let $x \in N R_{*}(M \cap Z)=\cup\{Y \in \psi: Y \subseteq(M \cap Z)\}$. There exists $Y \in \psi$ such that $x \in Y \subseteq$ $(M \cap Z), x \in Y \subseteq M$ and $x \in Y \subseteq Z$. Consequently, $x \in N R_{*} M$ and $x \in N R_{*} Z$, implying $x \in N R_{*} M$ $\cap N R_{*} Z$. Thus $N R_{*}(M \cap Z) \subseteq N R_{*} M \cap N R_{*} Z$.

(viii) Let $x \notin N R_{*}(M \cup Z)=\cup\{Y \in \psi: Y \subseteq M \cup Z\}$. For all $a \in M, x \in Y$, we have $Y \nsubseteq M \cup Z$, thus for all $a \in A, x \in Y$, we have $Y \nsubseteq M$ and $Y \nsubseteq Z Z$. Consequently, $x \notin N R_{*} M$ and $x \notin N R_{*} Z$, implying $x \notin N R_{*} M \cup N R_{*} Z$. Thus $N R_{*}(M \cup Z) \supseteq N R_{*} M \cup N R_{*} Z$.

(ix) Let $x \in N R^{*}(M \cap Z)=\cup\{Y \in \psi: Y \cap(M \cap Z) \neq \varnothing\}$. There exists $Y \in \psi$ such that $x \in Y$, $Y \cap(M \cap Z) \neq \varnothing, Y \cap M \neq \varnothing$ and $Y \cap Z \neq \varnothing$. Consequently, $x \in N R^{*} M$ and $x \in N R^{*} Z$, implying $x \in$ $N R^{*} M \cap N R^{*} Z$. Thus $N R^{*}(M \cap Z) \subseteq N R^{*} M \cap N R^{*} Z$.

(x) Let $x \notin N R^{*}(M \cup Z)=\cup\{Y \in \psi: Y \cap(M \cup Z) \neq \varnothing\}$. For all $a \in A, x \in Y$, we have $Y \cap(M \cup Z)=\varnothing$. For all $a \in A, x \in Y$, we have $Y \cap M=\varnothing$ and $Y \cap Z=\varnothing$. Consequently, $x \notin$ $N R^{*} M$ and $x \notin N R^{*} Z$, implying $x \notin N R^{*} M \cup N R^{*} Z$. Therefore, $N R^{*}(M \cup Z) \supseteq N R^{*} M \cup N R^{*} Z$. In addition, let $x \in N R^{*}(M \cup Z)=\cup\{Y \in \psi: Y \cap(M \cup Z) \neq \varnothing\}$, and thus, there exists $Y \in \psi$ such that $x \in Y, Y \cap(M \cup Z) \neq \varnothing$. It follows that, $Y \cap M \neq \varnothing$ or $Y \cap Z \neq \varnothing$. Consequently, $x \in N R^{*} M$ or $x \in$ $N R^{*} Z$. Hence, $x \in N R^{*} M \cup N R^{*} Z$, and $N R^{*} M \cup N R^{*} Z \supseteq N R^{*}(M \cup Z)$. Thus $N R^{*} M \cup N R^{*} Z=$ $N R^{*}(M \cup Z)$.

The converse of property (i) in Proposition 3 does not hold, as shown below.

Example 4. From Example 1, if $M=\left\{x_{1}, x_{4}\right\}$, then $N R_{*} M=\left\{x_{1}\right\}$ and $N R^{*} M=X$. Hence, $N R_{*} M \neq M$ and $M \neq N R^{*} M$.

The converse of property (iv) in Proposition 3 does not hold, as shown below.

Example 5. From Example 1, if $M=\left\{x_{2}\right\}$ and $Z=\left\{x_{1}, x_{2}\right\}$, then $N R_{*} M=\varnothing, N R_{*} Z=\left\{x_{1}, x_{2}\right\}$. Thus $N R_{*} M \neq N R_{*} Z$.

The converse of property (v) in Proposition 3 does not hold, as shown below.

Example 6. According to Example 1. Let $A=\left\{a_{1}\right\}$, then $\psi=\left\{\left\{x_{1}\right\},\left\{x_{5}\right\},\left\{x_{1}, x_{2}\right\},\left\{x_{1}, x_{3}\right\},\left\{x_{1}, x_{3}, x_{4}\right\}\right\}$. If $M=\left\{x_{2}\right\}$ and $Z=\left\{x_{1}, x_{2}\right\}$, then $N R^{*} M=\left\{x_{1}, x_{2}\right\}$ and $N R^{*} Z=\left\{x_{1}, x_{2}, x_{3}, x_{4}\right\}$. Hence, $N R^{*} M \neq$ $N R^{*} Z$.

The converse of property (vi) in Proposition 3 does not hold, as shown below.

Example 7. From Example 1, if $M=\left\{x_{1}, x_{3}, x_{4}\right\}$ and $Z=\left\{x_{1}, x_{4}, x_{5}\right\}$, then $N R_{*} M=\left\{x_{1}, x_{3}, x_{4}\right\}, N R_{*} Z$ $=\left\{x_{1}, x_{4}, x_{5}\right\}$ and $N R_{*}(M \cap Z)=\left\{x_{1}\right\}$. Hence, $N R_{*}(M \cap Z) \neq N R_{*} M \cap N R_{*} Z$.

The converse of property (vii) in Proposition 3 does not hold, as shown below.

Example 8. From Example 1, if $M=\left\{x_{1}\right\}$ and $Z=\left\{x_{2}\right\}$, then $N R_{*} M=\left\{x_{1}\right\}, N R_{*} Z=\varnothing$ and $N R_{*}(M \cup Z)$ $=\left\{x_{1}, x_{2}\right\}$. Hence, $N R_{*}(M \cup Z) \neq N R_{*} M \cup N R_{*} Z$.

The converse of property (viii) in Proposition 3 does not hold, as shown below.

Example 9. From Example 6, if $M=\left\{x_{2}, x_{5}\right\}$ and $Z=\left\{x_{1}, x_{3}, x_{5}\right\}$, then $N R^{*} M=\left\{x_{1}, x_{2}, x_{5}\right\}, N R^{*} Z=$ $X$ and $N R^{*}(M \cap Z)=\left\{x_{5}\right\}$. Hence, $N R^{*}(M \cap Z) \neq N R^{*} M \cap N R^{*} Z$. 
Proposition 4. Let $(H, A)$ be an (NSS) on a universe $X$, and let $M, Z \subseteq X$. Then the properties below hold.

(i) $N R_{*} N R_{*} M=N R_{*} M$.

(ii) $N R^{*} N R^{*} M \supseteq N R^{*} M$.

(iii) $N R_{*} N R^{*} M=N R^{*} M$.

(iv) $N R^{*} N R_{*} M \supseteq N R_{*} M$.

(v) $N R_{*} M^{c} \supseteq\left[N R^{*} M\right]^{c}$.

(vi) $N R^{*} M^{c} \supseteq\left[N R_{*} M\right]^{c}$.

Proof. (i) Let $W=N R_{*} M$ and $x \in W=\cup\{Y \in \psi: Y \subseteq M\}$. Then, for some $a \in A, x \in Y \subseteq W$. So, $x \in N R_{*} W$. Therefore, $W \subseteq N R_{*} W$. Hence $N R_{*} M \subseteq N R_{*} N R_{*} M$. From property (i) of Proposition 3, $N R_{*} M \subseteq M$ and using property (iv) of Proposition 3, we obtain $N R_{*} N R_{*} M \subseteq N R_{*} M$. Subsequently, $N R_{*} N R_{*} M=N R_{*} M$.

(ii) Let $W=N R^{*} M$. Using property (i) of Proposition 3, we get $W \subseteq N R^{*} W$. Hence $N R^{*} N R^{*} M$ $\supseteq N R^{*} M$.

(iii) Let $W=N R^{*} M$. Using property (i) of Proposition 3, we get $N R_{*} W \subseteq W$. Let $x \in W=$ $\cup\{Y \in \psi: Y \cap M \neq \varnothing\}$, thus there exists $Y \in \psi$ where $x \in Y \subseteq W$ such that $x \in N R_{*} W$. Subsequently, $W \subseteq N R_{*} W$, with $W=N R_{*} W$, and $W=N R^{*} M$. Therefore, $N R_{*} N R^{*} M=N R^{*} M$.

(iv) Let $W=N R_{*} M$. Using property (i) of Proposition 3, we get $W \subseteq N R^{*} W$. Hence $N R^{*} N R_{*} M$ $\supseteq N R_{*} M$.

(v) Let $x \notin N R_{*} M^{c}$. For all $Y \in \psi$ such that $x \in Y$, we have $Y \not \subset M^{c}$ and $Y \cap M^{c}=\varnothing$. Thus $Y \cap$ $M \neq \varnothing$, where $x \in N R^{*} M$ but $x \notin\left[N R^{*} M\right]^{c}$. Therefore, $N R_{*} M^{c} \supseteq\left[N R^{*} M\right]^{c}$.

(vi) From property (v) of Proposition 4, we get $N R_{*} M^{c} \supseteq\left[N R^{*} M\right]^{c}$.

Therefore, $N R_{*} M \supseteq\left[N R^{*} M^{c}\right]^{c}$ meaning that $N R^{*} M^{c} \supseteq\left[N R_{*} M\right]^{c}$.

The converse of property (ii) in Proposition 4 does not hold, as shown below.

Example 10. From Example 6, if $X=\left\{x_{2}\right\}$, we will have $N R^{*} X=\left\{x_{1}, x_{2}\right\}$ and $N R^{*} N R^{*} X=$ $\left\{x_{1}, x_{2}, x_{3}, x_{4}\right\}$. Therefore, $N R^{*} N R^{*} X \neq N R^{*} X$.

The converse of property (iv) in Proposition 4 does not hold, as shown below.

Example 11. From Example 6, if $M=\left\{x_{1}, x_{4}\right\}$, then $N R_{*} M=\left\{x_{1}\right\}$ and $N R^{*} N R_{*} M=\left\{x_{1}, x_{2}, x_{3}, x_{4}\right\}$. Hence, $N R^{*} N R_{*} M \neq N R_{*} M$.

The converse of property (v) in Proposition 4 does not hold, as shown below.

Example 12. From Example 6, if $M=\left\{x_{3}\right\}$, then $N R_{*} M^{c}=\left\{x_{1}, x_{2}, x_{5}\right\}$ and $\left[N R^{*} M\right]^{c}=\left\{x_{2}, x_{5}\right\}$. Hence, $N R_{*} M^{c} \neq\left[N R^{*} M\right]^{c}$.

The converse of property (vi) in Proposition 4 does not hold, as shown below.

Example 13. From Example 6, if $M=\left\{x_{1}, x_{2}, x_{4}, x_{5}\right\}$, then $\left[N R_{*} M\right]^{c}=\left\{x_{3}, x_{4}\right\}$ and $N R^{*} M^{c}=\left\{x_{1}, x_{3}, x_{4}\right\}$. Hence, $\left[N R_{*} M\right]^{c} \neq N R^{*} M^{c}$.

Proposition 5. Let $(H, A)$ be an (NSS) on a universe $X$, and let $M, Z \subseteq X$. Then,

$$
N R_{*}(M-Z) \subseteq N R_{*} M-N R_{*} Z \text {. }
$$

Proof. Let $u \in N R_{*}(M-Z)=\cup\{Y \in \psi: Y \subseteq(M-Z)\}$. There exists $Y \in \psi$ where $u \in Y \subseteq(M-Z)$, $u \in Y \subseteq M$ and $u \in Y \not Z Z$. Subsequently, $u \in N R_{*} M$ but $u \notin N R_{*} Z$, hence $u \in N R_{*} M-N R_{*} Z$. Thus, $N R_{*}(M-Z) \subseteq N R_{*} M-N R_{*} Z$. 
The converse of Proposition 5 does not hold, as shown below.

Example 14. From Example 1, if $M=\left\{x_{1}, x_{3}, x_{5}\right\}$ and $Z=\left\{x_{1}, x_{5}\right\}$, then $N R_{*} M=\left\{x_{1}, x_{3}, x_{5}\right\}, N R_{*} Z=$ $\left\{x_{1}, x_{5}\right\}, N R_{*}(M-Z)=\varnothing$ and $N R_{*} M-N R_{*} Z=\left\{x_{3}\right\}$. Hence, $N R_{*} M-N R_{*} Z \neq N R_{*}(M-Z)$.

Proposition 6. Let $(H, A)$ be an (NSS) on a universe $X$, and let $M, Z \subseteq X$. Then, the property below holds.

$$
N R^{*}(M-Z) \neq N R^{*} M-N R^{*} Z .
$$

Example 15. From Example 6, if $M=\left\{x_{1}, x_{3}, x_{5}\right\}$ and $Z=\left\{x_{1}, x_{5}\right\}$, then $N R^{*} M=X, N R^{*} Z=X$, $N R^{*}(M-Z)=\left\{x_{1}, x_{3}, x_{4}\right\}$ and $N R^{*} M-N R^{*} Z=\varnothing$. Hence, $N R^{*} M-N R^{*} Z \neq N R^{*}(M-Z)$.

\section{The Concepts of Neutrosophic Soft Rough Set}

We will now define the neutrosophic soft rough concepts as a generalization of rough concepts, illustrated by examples.

Definition 10. Let $(H, A)$ be an (NSS) on a universe $X$ and let $M \subseteq X$. A subset $M \subseteq X$ is called

(i) NSR-definable (NSR-exact) set, if $N R_{*} M=N R^{*} M=M$.

(ii) Internally NSR-definable set, if $N R_{*} M=M$ and $N R^{*} M \neq M$.

(iii) Externally NSR-definable set, if $N R_{*} M \neq M$ and $N R^{*} M=M$.

(iv) NSR-rough set, if $N R_{*} M \neq M$ and $N R^{*} M \neq M$.

Example 16. From Example 6, we have $\left\{x_{1}, x_{2}, x_{3}, x_{4}\right\}$ is NSR-definable set, whereas $\left\{x_{1}\right\},\left\{x_{5}\right\},\left\{x_{1}, x_{2}\right\}$, $\left\{x_{1}, x_{3}\right\},\left\{x_{1}, x_{5}\right\},\left\{x_{1}, x_{3}, x_{4}\right\},\left\{x_{1}, x_{3}, x_{5}\right\},\left\{x_{1}, x_{2}, x_{3}, x_{5}\right\},\left\{x_{1}, x_{3}, x_{4}, x_{5}\right\}$ are internally NSR-definable sets, whereas the rest of the subsets of $X$ are NSR-rough sets.

The degree of NSR-crispness (exactness) of any subset $M \subseteq X$, can be determined by using $N S R_{P}$-accuracy measure denoted by $C_{N S R} M$, which is defined as follows.

Definition 11. Let $(H, A)$ be an (NSS) on a universe $X$ and let $M \subseteq X$. Then,

$$
C_{N S R} M=\frac{\left|N R_{*} M\right|}{\left|N R^{*} M\right|}
$$

where $M \neq \phi$ and $|M|$ denotes the cardinality of sets.

Remark 2. Let $(H, A)$ be an (NSS) on a universe X. A subset $M \subseteq X$ is NSR-definable, if and only if, $C_{\text {NSR }} M=1$.

Neutrosophic soft rough (NSR)-membership function is defined below.

Definition 12. Let $(H, A)$ be an (NSS) on a universe $X$ and let $M \subseteq X$.

NSR-membership function of an element $m$ to a set $M$ denoted by $N_{M}(m)$ is defined as follows.

$$
N_{M}(m)=\frac{\left|m_{A} \cap M\right|}{\left|m_{A}\right|}
$$

where $S_{A}(m)=\cap\left\{S_{a}(m): a \in A\right\}$ and $S_{a}(m)$ is a neutrosophic right neighborhood defined in Definition 7.

Proposition 7. Let $(H, A)$ be an (NSS) on a universe $X, M \subseteq X$ and let $N_{M}(m)$ be the membership function defined in Definition 12. Then the properties below holds:

$$
N_{M}(m) \in[0,1]
$$


Proof. From Definition 12, we have $\phi \subseteq S_{A}(m) \cap M \subseteq S_{A}(m)$, then $0 \leq\left|S_{A}(m) \cap M\right| \leq\left|S_{A}(m)\right|$ and $0 \leq \frac{\left|S_{A}(m) \cap M\right|}{\left|S_{A}(m)\right|} \leq 1$, thus $N_{M}(m) \in[0,1]$.

Proposition 8. Let $(H, A)$ be an (NSS) on a universe $X$ and let $M \subseteq X$. Then,

$$
m \in M \Leftrightarrow N_{M}(m)=1
$$

Proof. Let $N_{M}(m)=1$, if and only if, $\frac{\left|S_{A}(m) \cap M\right|}{\left|S_{A}(m)\right|}=1$, if and only if, $\left|S_{A}(m) \cap M\right|=\left|S_{A}(m)\right|$, if and only if, $S_{A}(m) \subseteq M$. However, from Proposition 2, we have $R_{a}$ is a reflexive relation for all $a \in A$. Hence $m \in S_{a}(m), \forall a \in A$. It follows that $m \in S_{A}(m)$. Hence $m \in M$, if and only if, $N_{M}(m)=1$.

Proposition 9. Let $(H, A)$ be an (NSS) on a universe $X$ and let $M \subseteq X$. If $M_{1} \subseteq M_{2}$, then the properties below hold:

(i) $N_{M_{1}}(m) \leq N_{M_{2}}(m)$

(ii) $N_{N R_{*} M_{1}}(m) \leq N_{N R_{*} M_{2}}(m)$

(iii) $N_{N R^{*} M_{1}}(m) \leq N_{N R^{*} M_{2}}(m)$

Proof. (i) If $M_{1} \subseteq M_{2}$, it follows that $S_{A}(m) \cap M_{1} \subseteq S_{A}(m) \cap M_{2}$, then $\left|S_{A}(m) \cap M_{1}\right| \leq\left|S_{A}(m) \cap M_{2}\right|$ and $\frac{\left|S_{A}(m) \cap M_{1}\right|}{\left|S_{A}(m)\right|} \leq \frac{\left|S_{A}(m) \cap M_{2}\right|}{\left|S_{A}(m)\right|}$, thus $N_{M_{1}}(m) \leq N_{M_{2}}(m)$.

(ii) We get the proof directly from property (i) of Proposition 9 and property (iv) of Proposition 3.

(iii) We get the proof directly from property (ii) of Proposition 9 and property (v) of Proposition 3.

Proposition 10. Let $(H, A)$ be an (NSS) on a universe $X$ and let $M \subseteq X$, then the following properties hold:

(i) $N_{N R_{*} M}(m) \leq N_{M}(m)$

(ii) $N_{M}(m) \leq N_{N R^{*} M}(m)$

(iii) $N_{N R_{*} M}(m) \leq N_{N R^{*} M}(m)$

Proof. The proof of properties (i), (ii) and (iii) can be obtained directly from Propositions 3 and property (i) of Proposition 9.

Definition 13. Let $(H, A)$ be a (NSS) on a universe $X$ and let $m \in X, M \subseteq X$. NSR-membership relations, denoted by $\underline{\epsilon}_{N S R}$ and $\bar{\epsilon}_{N S R}$, are defined below.

$$
\begin{aligned}
& m \in_{N S R} M, \text { if } m \in N R_{*} M, \\
& m \bar{\epsilon}_{N S R} M, \text { if } m \in N R^{*} M .
\end{aligned}
$$

Proposition 11. Let $(H, A)$ be an (NSS) on a universe $X$ and let $m \in X, M \subseteq X$. Then,

$$
\begin{gathered}
\text { (i) } m \underline{\in}_{N S R} M \longrightarrow m \in M \\
\text { (ii) } m \Phi_{N S R} M \longrightarrow m \notin M
\end{gathered}
$$

Proof. Proof of (i) and (ii) follows directly from Definition 13 and Proposition 3.

The following example illustrates that the converse of properties (i) and (ii) in Proposition 11 do not hold.

Example 17. In Example 1, if $M=\left\{x_{2}, x_{5}\right\}$, then $N R_{*} M=\left\{x_{5}\right\}$ and $N R^{*} M=X$. Hence $x_{2} \notin_{N S R} M$, although $x_{2} \in M$ and $x_{3} \notin M$, although $x_{3} \bar{\epsilon}_{N S R} M$. 
Proposition 12. Let $(H, A)$ be an (NSS) on a universe $X$, and let $M \subseteq X$. Then the properties below hold:

$$
\begin{aligned}
& (i) m \underline{\in}_{N S R} M \longrightarrow N_{M}(m)=1 \\
& \left(\text { ii) } N_{M}(m)=1 \longrightarrow m \bar{\epsilon}_{N S R} M\right.
\end{aligned}
$$

Proof. The proof of properties (i) and (ii) can be obtained directly from Definition 13 and Propositions 11.

The converse of property (ii) in Proposition 12 does not hold, as shown below.

Example 18. In Example 1, if $M=\left\{x_{1}, x_{4}\right\}$, then $N R_{*} M=\left\{x_{1}\right\}$ and $S_{A}\left(x_{4}\right)=\left\{x_{4}\right\}$, it follows that $N_{M}\left(x_{4}\right)=\frac{\left|S_{A}\left(x_{4}\right) \cap M\right|}{\left|S_{A}\left(x_{4}\right)\right|}=\frac{\left|\left\{x_{4}\right\}\right|}{\left|\left\{x_{4}\right\}\right|}=1$, although $x_{4} \notin_{N S R} M$.

The converse of Proposition 13 does not hold, as shown below.

Example 19. In Example 1, if $M=\left\{x_{1}, x_{4}\right\}$, then $N R^{*} M=X$ and $S_{A}\left(x_{3}\right)=\left\{x_{1}, x_{3}\right\}$, it follows that $x_{3}$ $\bar{\epsilon}_{\text {NSR }} M$, although $N_{M}\left(x_{3}\right)=\frac{\left|\left\{x_{1}\right\}\right|}{\left|\left\{x_{1}, x_{3}\right\}\right|}=\frac{1}{2} \neq 1$.

Proposition 13. Let $(H, A)$ be an (NSS) on a universe $X$ and let $M \subseteq X$. Then,

$$
m \notin_{N S R} M \longrightarrow N_{M}(m)=0
$$

Proof. Let $m E_{N S R} M$, then $m \notin \in N R^{*} M$, also from Definition 9, we conclude that $S_{a}(m) \cap M=$ $\phi, \forall a \in A$, but $S_{A}(m)=\cap\left\{S_{a}(m): a \in A\right\}$. Thus $S_{A}(m) \cap M=\phi$ and $\left|S_{A}(m) \cap M\right|=0$, and hence $N_{M}(m)=0$.

The following example illustrates that the converse of Proposition 13 does not hold.

Example 20. In Example 1, if $M=\left\{x_{2}\right\}$, then $N R^{*} M=\left\{x_{1}, x_{2}\right\}$ and $S_{A}\left(x_{1}\right)=\left\{x_{1}\right\}$. It follows that $x_{1} \bar{\epsilon}_{N S R} M$, although $N_{M}\left(x_{1}\right)=\frac{\left|\left\{x_{1}\right\} \cap\left\{x_{2}\right\}\right|}{\left|\left\{x_{1}\right\}\right|}=0$.

Proposition 14. Let $(H, A)$ be an (NSS) on a universe $X$ and let $M \subseteq X$. Then,

(i) $N_{M}(m)=0 \longrightarrow m \notin M$

(ii) $N_{M}(m)=0 \longrightarrow m \notin_{N S R} M$

Proof. The proof of properties (i) and (ii) are straightforward and therefore are omitted.

The converse of property (i) in Proposition 14 does not hold, as shown below.

Example 21. In Example 1, if $M=\left\{x_{1}, x_{3}, x_{4}\right\}$, then $S_{A}\left(x_{2}\right)=\left\{x_{1}, x_{2}\right\}$ and $N_{M}\left(x_{2}\right)=\frac{\left|\left\{x_{1}\right\}\right|}{\left|\left\{x_{1}, x_{2}\right\}\right|}=\frac{1}{2} \neq 0$, although $x_{2} \notin M$.

The converse of property (ii) in Proposition 14 does not hold, as shown below.

Example 22. In Example 1, if $M=\left\{x_{1}, x_{4}, x_{5}\right\}$, then $N R_{*} M=\left\{x_{1}, x_{4}, x_{5}\right\}$ and $x_{2_{A}}=\left\{x_{1}, x_{2}\right\}$, it follows that $N_{M}\left(x_{2}\right)=\frac{\left|\left\{x_{1}\right\}\right|}{\left|\left\{x_{1}, x_{2}\right\}\right|}=\frac{1}{2} \neq 0$, although $x_{2} \notin_{N S R} M$.

Definition 14. Let $(H, A)$ be an (NSS) on a universe $X$ and let $M, Z \subseteq X$. NSR-inclusion relations, denoted by $\subseteq_{N S R}$ and $\vec{\subset}_{N S R}$, are defined as follows.

$$
M \subseteq_{N S R} Z \text {, if } N R_{*} M \subseteq N R_{*} Z,
$$




$$
M \vec{\subset}_{N S R} Z \text {, if } N R^{*} M \subseteq N R^{*} Z \text {. }
$$

Proposition 15. Let $(H, A)$ be an (NSS) on a universe $X$ and let $M, Z \subseteq X$. Then,

$$
M \subseteq Z \longrightarrow M \subseteq_{N S R} Z \text { and } M \vec{\subset}_{N S R} Z
$$

Proof. It can be directly obtained from Proposition 3.

The inverse of Proposition 15 does not hold, as shown below.

Example 23. In Example 6, if $M=\left\{x_{1}, x_{4}\right\}$ and $Z=\left\{x_{1}, x_{5}\right\}$, then $N R_{*} M=\left\{x_{1}\right\}, N R_{*} Z=\left\{x_{1}, x_{5}\right\}$, $N R^{*} M=\left\{x_{1}, x_{2}, x_{3}, x_{4}\right\}$ and $N R^{*} Z=X$. Hence, $M \subseteq_{N S R} Z$ and $M \vec{\subset}_{N S R} Z$, although $M \nsubseteq Z Z$.

Proposition 16. Let $(H, A)$ be an (NSS) on a universe $X$ and let $M, Z \subseteq X$. If $M \subseteq_{N S R} Z$, then the following properties hold:

(i) $N_{N R_{*} M}(m) \leq N_{N R_{*} Z}(m)$

(ii) $N_{N R_{*} M}(m) \leq N_{Z}(m)$

(iii) $N_{N R_{*} M}(m) \leq N_{N R^{*} Z}(m)$

Proof. The proof can be directly obtained from Definition 14 and Proposition 9.

Proposition 17. Let $(H, A)$ be an (NSS) on a universe $X$ and let $M, Z \subseteq X$. If $M \vec{\subset}_{N S R} Z$, then the properties below hold:

(i) $N_{N R^{*} M}(m) \leq N_{N R^{*} Z}(m)$

(ii) $N_{M}(m) \leq N_{N R^{*} Z}(m)$

(iii) $N_{N R_{*} M}(m) \leq N_{N R^{*} Z}(m)$

Proof. It can be directly obtained from Definition 14 and Proposition 9.

Definition 15. Let $(H, A)$ be an (NSS) on a universe $X$ and let $M, Z \subseteq X$. NSR-equality relations are defined as follows.

$$
\begin{aligned}
& M \widetilde{\sim}_{N S R} Z \text {, if } N R_{*} M=N R_{*} Z \text {, } \\
& M \simeq_{N S R} Z \text {, if } N R^{*} M=N R^{*} Z \text {, } \\
& M \approx_{N S R} Z \text {, if } M \widetilde{\sim}_{N S R} Z \text { and } M \simeq_{N S R} Z \text {. }
\end{aligned}
$$

The example below illustrates Definition 15.

Example 24. In Example 6, suppose $M_{1}=\left\{x_{2}\right\}, M_{2}=\left\{x_{3}\right\}, M_{3}=\left\{x_{1}, x_{2}\right\}, M_{4}=\left\{x_{1}, x_{4}\right\}, M_{5}=$ $\left\{x_{3}, x_{5}\right\}$ and $M_{6}=\left\{x_{4}, x_{5}\right\}$. Then, $N R_{*} M_{1}=N R_{*} M_{2}=\varnothing, N R^{*} M_{3}=N R^{*} M_{4}=\left\{x_{1}, x_{2}, x_{3}, x_{4}\right\}, N R_{*} M_{5}$ $=N R_{*} M_{6}=\left\{x_{5}\right\}$ and $N R^{*} M_{5}=N R^{*} M_{6}=\left\{x_{1}, x_{3}, x_{4}, x_{5}\right\}$. Consequently, $M_{1} \bar{\sim}_{N S R} M_{2}, M_{3} \simeq_{N S R} M_{4}$ and $M_{5} \approx_{N S R} M_{6}$.

Proposition 18. Let $(H, A)$ be an (NSS) on a universe $X$ and let $M, Z \subseteq X$. Then,

(i) $M \widetilde{\sim}_{N S R} N R_{*} M$

(ii) $M=Z \longrightarrow M \approx_{N S R} Z$

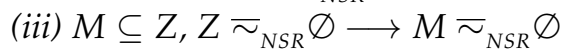

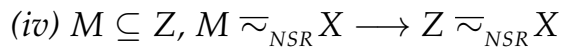

(v) $M \subseteq Z, Z \simeq_{N S R} \varnothing \longrightarrow M \simeq_{N S R} \varnothing$

(vi) $M \subseteq Z, M{\stackrel{N S R}{\simeq_{N S R}}} X Z{\stackrel{\sim}{\simeq_{N S R}}}^{\sim} X$

Proof. It can be directly obtained from Propositions 3 and 4 . 
Proposition 19. Let $(H, A)$ be an (NSS) on a universe $X$ and let $M, Z \subseteq X$. If $M \widetilde{\sim}_{N S R} Z$, then the following properties hold:

(i) $N_{N R_{*} M}(m)=N_{N R_{*} Z}(m)$

(ii) $N_{N R_{*} M}(m) \leq N_{Z}(m)$

(iii) $N_{N R_{*} M}(m) \leq N_{N R^{*} Z}(m)$

Proof. The proof of properties (i), (ii) and (iii) can be obtained directly from Definition 15 and Proposition 9.

Proposition 20. Let $(H, A)$ be an (NSS) on a universe $X$ and let $M, Z \subseteq X$. If $M \simeq_{N S R} Z$, then the following properties hold:

(i) $N_{N R^{*} M}(m)=N_{N R^{*} Z}(m)$

(ii) $N_{Z}(m) \leq N_{N R^{*} M}(m)$

(iii) $N_{N R_{*} Z}(m) \leq N_{N R^{*} M}(m)$

Proof. The proof of properties (i), (ii) and (iii) can be obtained directly from Definition 15 and Proposition 9.

\section{Application of the Proposed Neutrosophic Soft Rough Model in Decision Making}

This section presents an employment of the suggested neutrosophic soft rough approximations to the multi attribute decision making problems.

Consider Example 1 and suppose that we are requested to make a decision about the most desirable house based on the given attributes. To solve this problem, we apply the following decision steps.

Step 1: Input the NSS $(H, A)$.

Step 2: Compute the accuracy measure to each alternative (house) in the given $\operatorname{NSS}(H, A)$, separately.

Step 3: Choose the (element) alternative which has the highest accuracy measure as the optimal solution. If there is more than one alternative with highest accuracy measure, we do the following steps.

Step 4: Consider the alternatives that have the highest accuracy measure and create a new NSS $(\widehat{H}, A)$, which consists of the selected alternatives $x_{i}$ and the corresponding parameters $a_{j}$.

Step 5: $\quad$ Find the values of $S_{i j}=T_{a_{j}}\left(x_{i}\right)+I_{a_{j}}\left(x_{i}\right)-F_{a_{j}}\left(x_{i}\right)$, where $T, I$ and $F$ represent, respectively the truth, indeterminacy and falsity membership functions of the NSS $(\widehat{H}, A)$.

Step 6: Compute the score $C\left(x_{i}\right)=\sum_{j=1}^{m} S_{i j}$ of each element of the selected alternatives, where $m$ is the number of the parameters.

Step 7: Determine the value of the highest score. Then the decision is to choose the alternative with the highest score. If more than one alternative has the maximum score, then any one of those alternatives can be the optimal solution.

Table 2 gives the accuracy measure to all alternatives.

Table 2. Accuracy measures of the alternatives.

\begin{tabular}{cccccc}
\hline Alternatives & $x_{1}$ & $x_{2}$ & $x_{3}$ & $x_{4}$ & $x_{5}$ \\
\hline$C_{N S R} M$ & $\frac{1}{2}$ & 0 & 0 & 0 & $\frac{1}{2}$ \\
\hline
\end{tabular}


From Table 2, it is clear that there are two alternatives (houses) with the highest accuracy measure which are house 1 and house 5 . Thus, we proceed to the next steps and create the NSS $(\widehat{H}, A)$ of the considered alternatives as in Table 3.

Table 3. Tabular representation of $(\widehat{H}, A)$.

\begin{tabular}{ccccc}
\hline $\boldsymbol{X}$ & $\boldsymbol{a}_{1}$ & $\boldsymbol{a}_{2}$ & $\boldsymbol{a}_{3}$ & $\boldsymbol{a}_{4}$ \\
\hline$x_{1}$ & $(0.6,0.6,0.2)$ & $(0.8,0.4,0.3)$ & $(0.7,0.4,0.3)$ & $(0.8,0.6,0.4)$ \\
$x_{5}$ & $(0.8,0.2,0.3)$ & $(0.8,0.3,0.2)$ & $(0.7,0.3,0.4)$ & $(0.9,0.5,0.7)$ \\
\hline
\end{tabular}

Now we calculate the values of $S_{i j}$ to the considered alternatives (houses) as in the Table 4.

Table 4. Values of $S_{i j}$ to the considered houses.

\begin{tabular}{ccccc}
\hline $\boldsymbol{X}$ & $\boldsymbol{a}_{\mathbf{1}}$ & $\boldsymbol{a}_{\mathbf{2}}$ & $\boldsymbol{a}_{\mathbf{3}}$ & $\boldsymbol{a}_{\mathbf{4}}$ \\
\hline$x_{1}$ & 1 & 0.9 & 0.8 & 1 \\
$x_{5}$ & 0.7 & 0.9 & 0.6 & 0.7 \\
\hline
\end{tabular}

The scores $C\left(x_{i}\right)$ of the considered houses can be shown as in Table 5 .

Table 5. Scores of the considered houses.

\begin{tabular}{cc}
\hline $\boldsymbol{X}$ & Score \\
\hline$x_{1}$ & 3.7 \\
$x_{5}$ & 2.9 \\
\hline
\end{tabular}

From Table 5, it is clear that the house $x_{1}$ gets the highest score which is 3.7. Thus the decision is to choose house 1 as the appropriate solution under the parameter set $A$.

\section{Discussion}

We will discuss the features and limitations of our model by conducting a comparison with the existing models. Discussion will begin on the features of the proposed model before moving on to its limitations.

To illustrate the features of our model, we compare it with traditional rough approach $[5,33]$, neutrosophic rough set approaches [10,23-25], and fuzzy and intuitionistic fuzzy rough soft approaches [34-36].

We begin by making a comparison between the proposed neutrosophic soft rough approach and the traditional rough approach. The following Table 6 shows the properties of both traditional rough and the proposed neutrosophic soft rough approaches.

Table 6. Properties of traditional rough and neutrosophic soft rough.

\begin{tabular}{cc}
\hline Traditional Rough Properties & Neutrosophic Soft Rough Properties \\
\hline$\underline{E}(M \cap Z)=\underline{E}(M) \cap \underline{E}(Z)$ & $N R_{*}(M \cap Z) \subseteq N R_{*} M \cap N R_{*} Z$ \\
$\bar{E}(\bar{E}(M))=\bar{E}(M)$ & $N R^{*} N R^{*} M \supseteq N R^{*} M$ \\
$\bar{E}(\underline{E}(M))=\underline{E}(M)$ & $N R^{*} N R_{*} M \supseteq N R_{*} M$ \\
$\underline{E}\left(M^{c}\right)=[\bar{E}(M)]^{c}$ & $N R_{*} M^{c^{c}} \supseteq\left[N R^{*} M\right]^{c}$ \\
$\bar{E}\left(M^{c}\right)=[\underline{E}(M)]^{c}$ & $N R^{*} M^{c} \supseteq\left[N R_{*} M\right]^{c}$ \\
\hline
\end{tabular}

In the proposed neutrosophic soft rough approach, let us consider the NSS $(H, A)$ on the universe $X$, where $x \in X$ and $M \subseteq X$. If we consider the case where $T_{a}\left(x_{i}\right)>0.5$, then $a(x)=1$, otherwise $a(x)=0$. Thus, the neutrosophic right neighborhood of an element $x$ is replaced by the following 
equivalence class $[x]=\left\{x_{i} \in X: a\left(x_{i}\right)=a(x), a \in A\right\}$. Subsequently, the neutrosophic soft rough set approximates to that of Pawlak, i.e., the lower and upper approximations of the proposed model will be $N R_{*} M=\{x \in X:[x] \subseteq M\}$ and $N R^{*} M=\{x \in X:[x] \cap M \neq \varnothing\}$. Therefore, all properties of traditional rough set approximations will be satisfied.

We continue our discussion by comparing the proposed neutrosophic soft rough approach with other approaches which combine rough set to neutrosophic set [10,23-25]. It can be seen that these approaches have the inadequacy of the parametrization tool to facilitate the representation of parameters, while the soft set in the proposed model can represent the problem parameters in a more complete manner. This feature makes the proposed model superior to these models and other models that do not incorporate soft sets into their structures.

Now, we compare the proposed model to the fuzzy and intuitionistic fuzzy soft rough approaches [34-36]. The proposed approach combines rough set to neutrosophic soft set which is a generalisation of fuzzy and intuitionistic fuzzy soft set. Neutrosophic soft sets consider three membership functions instead of two as in the intuitionistic fuzzy soft set and one as in the fuzzy soft set. Fuzzy sets handle the uncertainty in data, intuitionistic fuzzy sets deal with ambiguous and incomplete data, while neutrosophic sets hold the features of all of the aforementioned sets in addition to its ability to handle the indeterminacy in data. Thus, combining neutrosophic soft sets to the rough sets provides the opportunity to deal with complicated data that cannot be handled by other models. From Example 1, it can be seen that fuzzy soft set and intuitionistic fuzzy soft set cannot describe the data presented by the neutrosophic soft set, which makes these models incapable to be applied directly on decision making problems with neutrosophic soft information. Conversely, the newly proposed model can directly address fuzzy and intuitionistic fuzzy soft rough set based decision making, since the intuitionistic fuzzy soft set is a special case of neutrosophic soft set and can be easily represented in the form of neutrosophic soft set. For example, the intuitionistic fuzzy soft value $(0.4,0.5)$ can be represented as $(0.4,0.1,0.5)$ by means of neutrosophic soft set, since the sum of the degrees of membership, nonmembership and indeterminacy of an intuitionistic fuzzy value equals to 1 . Note that the indeterminacy degree in intuitionistic fuzzy set is provided by default and cannot be defined alone unlike the neutrosophic set where the indeterminacy is defined independently and quantified explicitly.

Then we enlarge the discussion by presenting two limitations of the proposed model: (1) It cannot be used to solve multi attribute group decision making problems which incorporate the opinions of more than one expert. For more illustration, if we consider Example 1 and suppose that there are three experts who are requested to provide their opinions on each house under each (attribute) parameter, then we need a mechanism to incorporate the opinions of the three experts in one model (neutrosophic soft set), otherwise, we have to construct three neutrosophic soft sets and this increases the amount of both mathematical calculations and investigation of several operators in incorporating three neutrosophic soft sets to find out the optimal solution; (2) There exist some neutrosophic soft set based decision making problems in which the proposed algorithm is likely to get an empty decision (optimum) set. Consider Example 1, and consider the NSS $(G, A)$ as in Table 7.

Table 7. Tabular representation of the NSS $(G, A)$.

\begin{tabular}{ccccc}
\hline $\boldsymbol{X}$ & $\boldsymbol{a}_{1}$ & $\boldsymbol{a}_{2}$ & $\boldsymbol{a}_{3}$ & $\boldsymbol{a}_{\mathbf{4}}$ \\
\hline$x_{1}$ & $(0.6,0.6,0.2)$ & $(0.8,0.4,0.3)$ & $(0.7,0.4,0.3)$ & $(0.8,0.6,0.4)$ \\
$x_{2}$ & $(0.4,0.6,0.6)$ & $(0.6,0.2,0.4)$ & $(0.6,0.4,0.3)$ & $(0.7,0.6,0.6)$ \\
$x_{3}$ & $(0.6,0.4,0.2)$ & $(0.8,0.2,0.2)$ & $(0.7,0.4,0.3)$ & $(0.7,0.6,0.4)$ \\
$x_{4}$ & $(0.6,0.3,0.3)$ & $(0.8,0.2,0.2)$ & $(0.5,0.2,0.6)$ & $(0.7,0.5,0.6)$ \\
$x_{5}$ & $(0.6,0.6,0.2)$ & $(0.8,0.4,0.3)$ & $(0.7,0.3,0.4)$ & $(0.8,0.6,0.4)$ \\
\hline
\end{tabular}

We then obtain the family of all neutrosophic right neighborhood $\psi=\left\{\left\{x_{1}, x_{3}\right\},\left\{x_{1}, x_{5}\right\}\right.$, $\left.\left\{x_{1}, x_{2}, x_{3}\right\},\left\{x_{1}, x_{3}, x_{5}\right\},\left\{x_{1}, x_{2}, x_{3}, x_{5}\right\},\left\{x_{1}, x_{3}, x_{4}, x_{5}\right\},\left\{x_{3}, x_{4}\right\}, X\right\}$.

As a result, the accuracy measure table is as follows. 
From Table 8, it can be seen that the accuracy measure to each alternative (house) equals zero, which means that none of the houses can be selected as a candidate to be an optimal solution. Thus the proposed approach fails to handle this case.

Table 8. Accuracy measures of the alternatives in the NSS $(G, A)$.

\begin{tabular}{cccccc}
\hline Alternatives & $x_{1}$ & $x_{2}$ & $x_{3}$ & $x_{4}$ & $x_{5}$ \\
\hline$C_{N S R} M$ & 0 & 0 & 0 & 0 & 0 \\
\hline
\end{tabular}

\section{Conclusions}

We proposed a novel approach to rough sets based on neutrosophic soft sets and deduced that the traditional rough approach is a special case of the proposed approach. The lower and upper neutrosophic soft rough approximations are defined and their properties are verified. We have further defined some essential neutrosophic soft rough concepts such as neutrosophic soft rough (NSR) definability, neutrosophic soft rough (NSR)-membership relations and functions. Properties of these concepts are deduced, proven and shown by several examples. In addition, we provided an algorithm based on the proposed neutrosophic soft rough sets approximations. Finally, we have made a comparative analysis and a discussion to reveal the features and limitations of the proposed model. For the future prospects, we will extend this model by using topological structures and commit to exploring the application of the proposed model to data mining and attribute reduction.

Author Contributions: E.M. proposed the concept of neutrosophic right neighborhood; A.A.-Q. and N.H. studied the neutrosophic soft rough set approximations and their properties; A.A.-Q. introduced some concepts on neutrosophic soft rough set and provided some illustrated examples; N.H. edited the paper and all authors wrote it.

Funding: This research was funded by Universiti Kebangsaan Malaysia GUP-2017-105.

Acknowledgments: We are indebted to Universiti Kebangsaan Malaysia for providing financial support and facilities for this research under the grant GUP-2017-105.

Conflicts of Interest: Authors declare that they have no conflict of interest.

\section{References}

1. Zadeh, L.A. Fuzzy sets. Inf. Control 1965, 8, 338-353. [CrossRef]

2. Atanassov, K. Intuitionistic fuzzy sets. Fuzzy Sets Syst. 1986, 20, 87-96. [CrossRef]

3. Gau, L.W.; Buehrer, D.J. Vague sets. IEEE Trans Syst Man Cybern. 1993, 23, 610-614. [CrossRef]

4. Gorzalzany, M.B. A method of inference in approximate reasoning based on interval-valued fuzzy sets. Fuzzy Sets Syst. 1987, 21, 1-17. [CrossRef]

5. Pawlak, Z. Rough sets. Int. J. Comput. Inf. Sci. 1982, 11, 341-356. [CrossRef]

6. Slowinski, R.; Vanderpooten, D. A generalized definition of rough approximations based on similarity. IEEE Trans. Knowl. Data Eng. 2000, 12, 331-336. [CrossRef]

7. Greco, S.; Matarazzo, B.; Slowinski, R. Rough approximation of a preference relation by dominance relations. Eur. J. Oper. Res. 1999, 117, 63-83. [CrossRef]

8. Skowron, A.; Stepaniuk, J. Tolerance approximation spaces. Fundamen. Inform. 1996, 27, 245-253.

9. Greco, S.; Matarazzo, B.; Slowinski, R. Rough approximation by dominance relation. Int. J. Intell. Syst. 2002, 17, 153-171. [CrossRef]

10. Wang, J.; Zhang, X. Two types of single valued neutrosophic covering rough sets and an application to decision making. Symmetry 2018, 10, 710. [CrossRef]

11. Yao, Y.Y. Relational interpretation of neighborhood operators and rough set approximation operator. Inf. Sci. 1998, 111, 239-259. [CrossRef]

12. Molodtsov, D. Soft set theory: First results. Comput. Math. Appl. 1999, 37, 19-31. [CrossRef]

13. Maji, P.K.; Roy, A.R.; Biswas, R. An application of soft sets in a decision making problem. Comput. Math. Appl. 2002, 44, 1077-1083. [CrossRef]

14. Maji, P.K.; Biswas, R.; Roy, A.R. Fuzzy soft set theory. J. Fuzzy Math. 2001, 3, 589-602. 
15. Feng, F.; Liu, X.; Fotea, V.L.; Jun, Y.B. Soft sets and soft rough sets. Inf. Sci. 2011, 181, 1125-1137. [CrossRef]

16. Smarandache, F. Neutrosophy: Neutrosophic Probability, Set, and Logic; American Research Press: Rehoboth, IL, USA, 1998.

17. Smarandache, F. Neutrosophic set-A generalisation of the intuitionistic fuzzy sets. Int. J. Pure Appl. Math. 2005, 24, 287-297.

18. Smarandache, F. Neutrosophic Overset, Neutrosophic Underset, and Neutrosophic Offset. Similarly for Neutrosophic over-/under-/off-Logic, Probability, and Statistics; Pons Editions: Brussels, Belgium, 2016.

19. Jun, Y.B.; Smarandache, F.; Kim, C.S. Neutrosophic cubic sets. New Math. Nat. Comput. 2017, 13, 41-54. [CrossRef]

20. Maji, P.K. Neutrosophic soft set. Ann. Fuzzy Math. Inform. 2013, 5, 157-168.

21. Abu Qamar, M.; Hassan, N. Generalized Q-neutrosophic soft expert set for decision under uncertainty. Symmetry 2018, 10, 621. [CrossRef]

22. Ulucay, V.; Sahin, M.; Hassan, N. Generalized neutrosophic soft expert set for multiple-criteria decision-making. Symmetry 2018, 10, 437. [CrossRef]

23. Broumi, S.; Smarandache, F.; Dhar, M. Rough neutrosophic sets. Neutrosophic Sets Syst. 2014, 3, $62-67$.

24. Bo, C.; Zhang, X.; Shao, S.; Smarandache, F. Multi-granulation neutrosophic rough sets on a single domain and dual domains with applications. Symmetry 2018, 10, 296. [CrossRef]

25. Bo, C.; Zhang, X.; Shao, S.; Smarandache, F. New multigranulation neutrosophic rough set with applications. Symmetry 2018, 10, 578. [CrossRef]

26. Broumi, S.; Smarandache, F. Interval-valued neutrosophic soft rough sets. Int. J. Comput. Math. 2015, 2015, 232919 . [CrossRef]

27. Al-Quran, A.; Hassan, N. Neutrosophic vague soft set and its applications. Malays. J. Math. Sci. 2017, 11, 141-163.

28. Al-Quran, A.; Hassan, N. Neutrosophic vague soft multiset for decision under uncertainty. Songklanakarin J. Sci. Technol. 2018, 40, 290-305.

29. Ali, M.; Smarandache, F. Complex neutrosophic set. Neural Comput. Appl. 2017, 28, 1817-1834. [CrossRef]

30. Al-Quran, A.; Alkhazaleh, S. Relations between the complex neutrosophic sets with their applications in decision making. Axioms 2018, 7, 64. [CrossRef]

31. Al-Quran, A.; Hassan, N. The complex neutrosophic soft expert relation and its multiple attribute decision-making method. Entropy 2018, 20, 101. [CrossRef]

32. Quek, S.G.; Broumi, S.; Selvachandran, G.; Bakali, A.; Talea, M.; Smarandache, F. Some results on the graph theory for complex neutrosophic sets. Symmetry 2018, 10, 190. [CrossRef]

33. Pawlak, Z.; Skowron, A. Rudiments of rough sets. Inf. Sci. 2007, 177, 3-27. [CrossRef]

34. Zhang, Z. A rough set approach to intuitionistic fuzzy soft set based decision making. Appl. Math. Model. 2012, 36, 4605-4633. [CrossRef]

35. Zhang, H.; Shu, L.; Liao, S. Intuitionistic fuzzy soft rough set and its application in decision making. Abstr. Appl. Anal. 2014, 2014, 287314. [CrossRef]

36. Meng, D.; Zhang, X.; Qin, K. Soft rough fuzzy sets and soft fuzzy rough sets. Comput. Math. Appl. 2011, 26, 4635-4645. [CrossRef]

(C) 2019 by the authors. Licensee MDPI, Basel, Switzerland. This article is an open access article distributed under the terms and conditions of the Creative Commons Attribution (CC BY) license (http://creativecommons.org/licenses/by/4.0/). 